\title{
EFFECT OF N-3 FATTY ACIDS SUPPLEMENTATION DURING LIFE STYLE MODIFICATION IN WOMEN WITH OVERWEIGHT
}

\author{
Pavel Sedláček1, Iveta Plavinová', Jana Langmajerová1, Jana Dvoráḱková1, Jaroslav Novák², Ladislav Trefil’3, \\ Luděk Müller', Petra Buňatová ${ }^{5}$, Václav Zeman², Dana Müllerová ${ }^{4}$ \\ 'Department of Public Health and Preventive Medicine, Faculty of Medicine in Pilsen, Charles University, Pilsen, Czech Republic \\ ${ }^{2}$ Department of Physical Education, Faculty of Medicine in Pilsen, Charles University, Pilsen, Czech Republic \\ ${ }^{3}$ Department of Clinical Biochemistry and Haematology, Faculty of Medicine and Faculty Hospital in Pilsen, Charles University, Pilsen, Czech \\ Republic \\ ${ }^{4}$ European Centre of Excellence, New Technologies for the Information Society, University of West Bohemia, Pilsen, Czech Republic \\ ${ }^{5}$ Department of Psychiatry, Faculty of Medicine in Pilsen, Charles University, Pilsen, Czech Republic
}

\section{SUMMARY}

Objective: The marine n-3 fatty acids eicosapentaenoic acid (EPA) and docosahexaenoic acid (DHA) exert numerous beneficial effects on health, but their potency to defend against development of peripheral insulin resistance of healthy person with overweight remains poorly characterized. We aimed to evaluate the effect of a combination intervention using EPA+DHA and the lifestyle modification (LSM) in women with overweight.

Method: In a parallel-group, three-arm, randomized trial (UMIN Clinical Trials Registry - R000031131), 34 women were assigned to a 12-weekintervention using corn oil (1.5 g/day; placebo); LSM and corn oil (1.5 g/day; LSM); or LSM and EPA+DHA concentrate (1.5 g/day, containing 0.6 g EPA+DHA; LSM \& n-3). At baseline and after intervention, anthropometric measurements including bioelectrical impedance analysis, spiroergometry, 24-hours dietary recall, and various metabolic markers, adiponectin and cytokines were evaluated in serum using standard procedures. Data from 29 women were used for the final evaluation. Wilcoxon two-sided rank-sum test was used to inspect the differences between LSM and LSM \& n-3, and placebo groups, with a p-value of $\leq 0.05$. All computations were performed with MATLAB Statistics Toolbox.

Results: In comparison with placebo, LSM and LSM \& n-3 decreased body weight, waist circumference, and body fat, and increased VO $\mathrm{max}_{2} / \mathrm{kg}$. LSM \& n-3 increased adiponectin levels in comparison to LSM. Fasting insulin, IL8, and cholesterol were decreased by LSM, but were unchanged by LSM \& n-3. IL6 was not affected in LSM \& n-3, while it was increased in LSM. Other inflammatory markers, as well as leptin, LIF, follistatin, $\mathrm{BDNF}$, and fasting triacylglycerol were not significantly affected by any of the interventions.

Conclusion: Besides preventing a modest negative effect of LSM on IL6 and adiponectin level, the combination of LSM and EPA+DHA supplementation could be probably used to improve the functional capacity of adipose tissue in women with overweight.

Key words: overweight, n-3 fatty acids, diet, physical activity, adiponectin

Address for correspondence: D. Müllerová, Department of Public Health and Preventive Medicine, Faculty of Medicine in Pilsen, Charles University, Lidická 4, 30166 Pilsen, Czech Republic. E-mail: dana.mullerova@lfp.cuni.cz

https://doi.org/10.21101/cejph.a5259

\section{INTRODUCTION}

Global obesity rates have doubled between 1980 and today. Overweight as defined by body mass index $(25 \leq \mathrm{BMI}<30 \mathrm{~kg} /$ $\mathrm{m}^{2}$ ) occurs in the magnitude of $20-40 \%$ in most European adult populations (1). Overweight represents a risk factor for the development of obesity and/or disorders associated with obesity. Physical inactivity and unhealthy nutrition increase risk of obesity and metabolic disturbances like insulin resistance and type 2 diabetes (T2D) (2) and vice versa, loss of weight excess with regular physical activity ameliorates glycaemic control (3-5). Lifestyle modification (LSM) may interfere with the complex aetiology of T2D and cardiovascular disease to target multiple underlying mechanisms. Indeed, the World Health Organization (WHO) has set standards for prevention of non-communicable disease concerning diet and physical activity (6). Major effects of regular physical activity include the lowering of fasting glycaemia and insulinemia and reduced risk of insulin resistance $(7,8)$. Physical activity shows as an independent anti-inflammatory action and amelioration of the oxidative stress (9). The effects of exercise however vary depending on the type, intensity, frequency, and duration of exercise as well as on the individual's characteristics; therefore, the development of personalized exercise programmes is essential (10). Moreover, regular physical activity decreases adiposity and protects fat free mass. On the other hand, enlarged white adipose tissue with morphological changes plays a key role in the development of systemic insulin resistance. The resistance is precipitated by impaired adipose tissue glucose and lipid metabolism, linked to a low-grade inflammation of adipose tissue and secretion of pro-inflammatory adipokines like tumor 
necrosis factor (TNF) and IL $6(11,12)$. Simultaneously, there is compromised secretion of adiponectin. Adiponectin, an abundant adipocyte-secreted factor with a wide-range of biological activities, improves insulin sensitivity in major insulin target tissues, modulates inflammatory responses, and plays a crucial role in the regulation of energy metabolism (12). Unlike most other adipocyte-derived hormones, adiponectin gene expression and blood concentration are inversely associated with adiposity (13). Development of metabolic syndrome with insulin resistance can be delayed, defended, or reversed by the combination of regular physical activity and healthy diet. Especially in the diet naturally occurring long-chain n-3 polyunsaturated fatty acids (PUFA), namely eicosapentaenoic acid (EPA; 20:5 n-3) and docosahexaenoic acid (DHA; 22:6n-3) exert anti-inflammatory and hypolipidemic effects (14). Regarding the effects of EPA+DHA on glycaemic control and insulin sensitivity, positive results have been obtained in animal models; however, mixed results were obtained with respect to prevention of type 2 diabetes mellitus by EPA+DHA in adults $(15,16)$.

In this study, we examined whether EPA+DHA supplementation at a relatively low dose of $\sim 0.6 \mathrm{~g}$ /day could modulate the effects of LSM in healthy women with overweight. The major goal of the study was to characterize the effect of the combined intervention on modulation of adipose tissue function.

\section{MATERIALS AND METHODS}

A 12-week, parallel-group, three-arm, randomized trial (UMIN Clinical Trials Registry - R000031131) was conducted in accordance with the principles of the Declaration of Helsinki (2008 revision) and with approval by the Institutional Ethical Committee. The study subjects were enrolled by mass media call requiring adult women, aged 20-60 years, with overweight, without chronic illness aiming to loose body weight. Women who did not want to adhere to life style modification (LSM) protocol were separated and included to the placebo group. All participants agreed and subscribed written informed consent before participation in the study. Inclusion criteria were 20-60 years of age, women, fulfilling two of three criteria for overweight: body mass index (BMI) in the range of $25-29.9 \mathrm{~kg} / \mathrm{m}^{2}$, waist circumference, equal or higher than $80 \mathrm{~cm}$, body fat measured by bioelectrical impedance analysis (BIA) equal or higher than $25 \%$ of total body weight; ability and willingness to adhere to the protocol and signed and dated written informed consent obtained before the study. Exclusion criteria were pregnancy and lactation, smoking, long-term medication, history of cardiovascular, endocrine, and metabolic diseases, cancer, significant recent weight gain or loss ( $>5 \%$ of total body weight within the past 3 months). Out of 52 responding women to a call to study in mass media subjected to initial screening, 34 eligible participants were enrolled. Data from 29 participants could be used for the final evaluation. Two participants withdrew owing to personal reasons; and after the intervention started, 3 participants were excluded due to failure to adhere to the study protocol. Study subjects were enrolled and LSM was controlled at the Department of Public Health and Preventive Medicine, Faculty of Medicine in Plzen. Participants were randomized to three groups: $1.5 \mathrm{~g}$ /day corn oil (placebo); LSM and corn oil (1.5 g/day; LSM); and LSM and EPA+DHA concentrate $(1.5 \mathrm{~g} /$ day, containing $\sim 0.6 \mathrm{~g}$ EPA+DHA, LSM \& n-3). Supplementation of EPA and DHA was provided by daily use of EPAMAR caps, Hankintatukku Ltd, containing about 33\% EPA, 21\% DHA, wt/wt; i.e., $\sim 0.6$ g EPA + DHA. Randomization between LSM and LSM \& n-3 was performed using a computerbased algorithm arranging experimental units in blocks of two. The randomization code was kept secret and revealed after the clean-file procedure had been completed when all data had been filled in the case report forms.

For each group, the trial was conducted in three phases (screening - week 0 , the intervention phase - weeks 1-12, final screening - week 13). All measurements, procedures and sample collections were performed at week 0 (baseline) and week 13 on an outpatient basis, after overnight $(8-10 \mathrm{~h})$ fasting. Serum samples were collected, anthropometric measurements including measurement of body fatness by BIA, and standard spiroergometry were performed. Habitual dietary intake at baseline and at the end of study were assessed by semi quantitative, 56 -item food frequency questionnaire (56-FFQ) and similarly participants were asked to record 24-hour dietary records.

LSM consisted of a 3-month complex self-tailored programme of defined and supervised physical activity and diet. Physical activity included 30 minutes of continuous walking from the total minimum amount of $>10,000$ steps a day (monitored by pedometers) and twice a week 1.5 hour of controlled and continuously monitored physical activity, where part of aerobic activity respected the initial physical fitness, measured by spiroergometry, continued to grow and was directed according to Karvonen's heart rate reserve method (17) and Borg's scale (18) of subjective perception of the intensity of the load in aerobic zone load ( $50-75 \%$ of their individual oxygen consumption reserve $-\mathrm{VO}_{2 \mathrm{R}}$ ) for 45 minutes on training simulators. Personalized diet recommendations in LSM and LSM \& n-3 groups were developed on the basis of energy expenditure assessment in combination with habitual caloric intake assessment. Dietary counselling was individualized for each person and participants were encouraged not to reduce caloric intake under the habitual amount, but rather to increase intake of vegetables, fruits, legumes, seeds, nuts, and fiber-rich complex carbohydrates, vegetable oils with monounsaturated fatty acids (MUFA), fish and low fat dairy products; and decrease intake of saturated (SFA) and trans fat, salt, sugars, and red meat. They were instructed to limit intake of vegetable oils with polyunsaturated $n-6$ fatty acids and exclude highly processed food from the diet as much as possible. Based on recommended energy content, the participants obtained framework diet plans and were initially educated and checked every week in required diet by dieticians.

Body composition was measured by tetra-polar and ipsilateral BIA (Nutriguard M, Data Input GmbH, Germany).

During spiroergometry (ergometer ER 900 Jaeger, heart rate monitor Polar, Finland) oxygen uptake $\left(\mathrm{VO}_{2}\right), \mathrm{CO}_{2}$ expenditure $\left(\mathrm{VCO}_{2}\right)$, minute ventilation (VE) and respiratory exchange ratio (R) were measured. The achievement of maximal oxygen uptake $\left(\mathrm{VO}_{2} \max \right)$ was based on objective criteria such as a levelling off of oxygen uptake in the final phase of the stress test, the elevated respiratory exchange ratio above 1.05 and achievement of an ageadjusted estimate maximal heart rate (19). The obtained values were computerized with help of the software Konsil (Stork, M 2001, Czech Republic). 
Blood samples for the serum analysis were prepared at the test facility by centrifuging at 3,600/min for 8 minutes; serum was then separated into aliquots and stored at $-80^{\circ} \mathrm{C}$ until analysis after the study was completed. Concentrations of serum cytokines were measured using ELISA kits from Ray Bio (Ray Biotech, GA) - total adiponectin, leptin, insulin, follistatin, brain-derived neurotrophic factor (BDNF), leukocyte inhibitory factor (LIF), tumor necrosis factor $\alpha$ (TNF $\alpha$ ), and interleukins (IL6, IL8). Vitamin D (25-OH vitamin D) was measured using ELISA kits from Biovendor (Czech Republic). Data from the baseline and the end of the study, and changes $(\Delta)$ between the baseline and the end of the study were considered for the statistical analyses. Neither the analyzed data nor their logarithmic (shifted) values were normally distributed, and Wilcoxon two-sided rank-sum test was used to inspect the differences between LSM and LSM \& $\mathrm{n}-3$, and control placebo groups. Threshold of significance was defined at a $p$ value of $\leq 0.05$. All computations were performed with MATLAB Statistics Toolbox.

\section{RESULTS}

No significant differences were observed between the subgroups in basic anthropometric and biochemical characteristics measured in the fasting state at the beginning of the study (Table 1). During trial we did not see any harm or unintended effects in the participants. Characteristics of diet and physical activity during trial in all subgroups are summarised in Tables 2 and 3. The effect of the 12-week-intervention in the subgroup analysis is described in Fig. 2 and in Table 4 (all values are presented as median and values of the first and third quartiles). LSM and LSM \& n-3 decreased body weight, waist circumference, body fat, and increased $\mathrm{VO}_{2}$ max $/ \mathrm{kg}$ (maximal oxygen consumption per $1 \mathrm{~kg}$ of weight) relative to baseline. LSM \& n-3 had increased adiponectin levels in comparison to LSM $(p<0.05)$ (Fig. 2). Fasting insulin, IL8, and total cholesterol were decreased by LSM, but were unchanged by LSM \& n-3. IL6 was not affected in LSM \& n-3, while it was increased in LSM (p<0.05) (Fig. 2). Other inflammatory markers, leptin, LIF, follistatin, BDNF, and fasting triacylglycerol were not significantly affected by any of the interventions (Table 4).

\section{DISCUSSION}

We show here that a 3-month-combined lifestyle intervention using moderate physical activity with iso-caloric diet and the supplementation of relatively low dose of EPA+DHA exerts during the weight- and fat-loss process additive beneficial effects on the adipose tissue endocrine function and systemic inflammation of both adiponectin and IL6 in women with overweight. Our results are in consistency with the finding of Ortega et al. (20) showing enhanced training effects in patients with metabolic syndrome under dietary supplementation with n-3 polyunsaturated fatty

Table 1. Baseline data, time 0

\begin{tabular}{|l|c|c|c|c|}
\hline \multirow{2}{*}{} & \multirow{2}{*}{ Unit } & Placebo & LSM & LSM \& omega 3 \\
\cline { 3 - 5 } & & Mean \pm SD & 10 & Mean \pm SD \\
\hline Age & - & 8 & $36.4 \pm 10.2$ & $42.6 \pm 9.8$ \\
\hline Weight & year & $51.4 \pm 10.8$ & $75.9 \pm 5.3$ & $72.9 \pm 5.7$ \\
\hline Body mass index & $\mathrm{kg} / \mathrm{m}^{2}$ & $77.5 \pm 5.9$ & $27.6 \pm 1.4$ & $26.3 \pm 1.0$ \\
\hline Waist circumference & $\mathrm{cm}$ & $28.0 \pm 1.0$ & $86.1 \pm 8.4$ & $83.1 \pm 4.8$ \\
\hline Hip circumference & $\mathrm{cm}$ & $90.6 \pm 7.4$ & $106.8 \pm 4.5$ & $105.2 \pm 4.2$ \\
\hline Leg circumference & $\mathrm{cm}$ & $106.4 \pm 4.3$ & $57.8 \pm 1.8$ & $56.7 \pm 2.8$ \\
\hline Arm circumference & $\mathrm{cm}$ & $56.9 \pm 3.6$ & $29.7 \pm 1.8$ & $28.9 \pm 3.3$ \\
\hline BIA body fat & $\%$ & $30.1 \pm 1.5$ & $31.1 \pm 2.3$ & $29.0 \pm 3.5$ \\
\hline BIA body cell mass & $\mathrm{kg}$ & $31.6 \pm 3.0$ & $28.2 \pm 2.8$ & $26.9 \pm 2.4$ \\
\hline BIA phase angle & $\circ$ & $27.7 \pm 2.5$ & $6.4 \pm 0.5$ & $6.2 \pm 0.5$ \\
\hline VO ${ }_{2}$ max & $\mathrm{L} / \mathrm{min}$ & $6.1 \pm 0.4$ & $31.7 \pm 5.5$ & $31.7 \pm 4.2$ \\
\hline W170/kg & - & $27.6 \pm 4.1$ & $1.4 \pm 0.2$ & $1.6 \pm 0.2$ \\
\hline METs & $\mathrm{MET}$ & $1.7 \pm 0.4$ & $11.2 \pm 1.5$ & $11.2 \pm 1.3$ \\
\hline Wmax/kg & - & $10.4 \pm 1.5$ & $2.1 \pm 0.4$ & $2.2 \pm 0.4$ \\
\hline Systolic blood pressure & $\mathrm{mmHg}$ & $1.7 \pm 0.4$ & $114.1 \pm 9.8$ & $117.3 \pm 11.2$ \\
\hline Diastolic blood pressure & $\mathrm{mmHg}$ & $124.3 \pm 10.4$ & $75.0 \pm 7.9$ & $76.2 \pm 8.3$ \\
\hline
\end{tabular}

LSM - group with placebo and managed physical activity

LSM \& omega 3 - group with n-3 PUFA and managed physical activity

$\mathrm{SD}$ - standard deviation

BIA - bioelectrical impedance analysis

$\mathrm{VO}_{2}$ max - maximal oxygen consumption

W170/kg - physical performance during heart rate $170 /$ minute related to $1 \mathrm{~kg}$ of body weight

MET - metabolic equivalent of task

Wmax $/ \mathrm{kg}$ - maximal physical performance per $\mathrm{kg}$ of body weight 


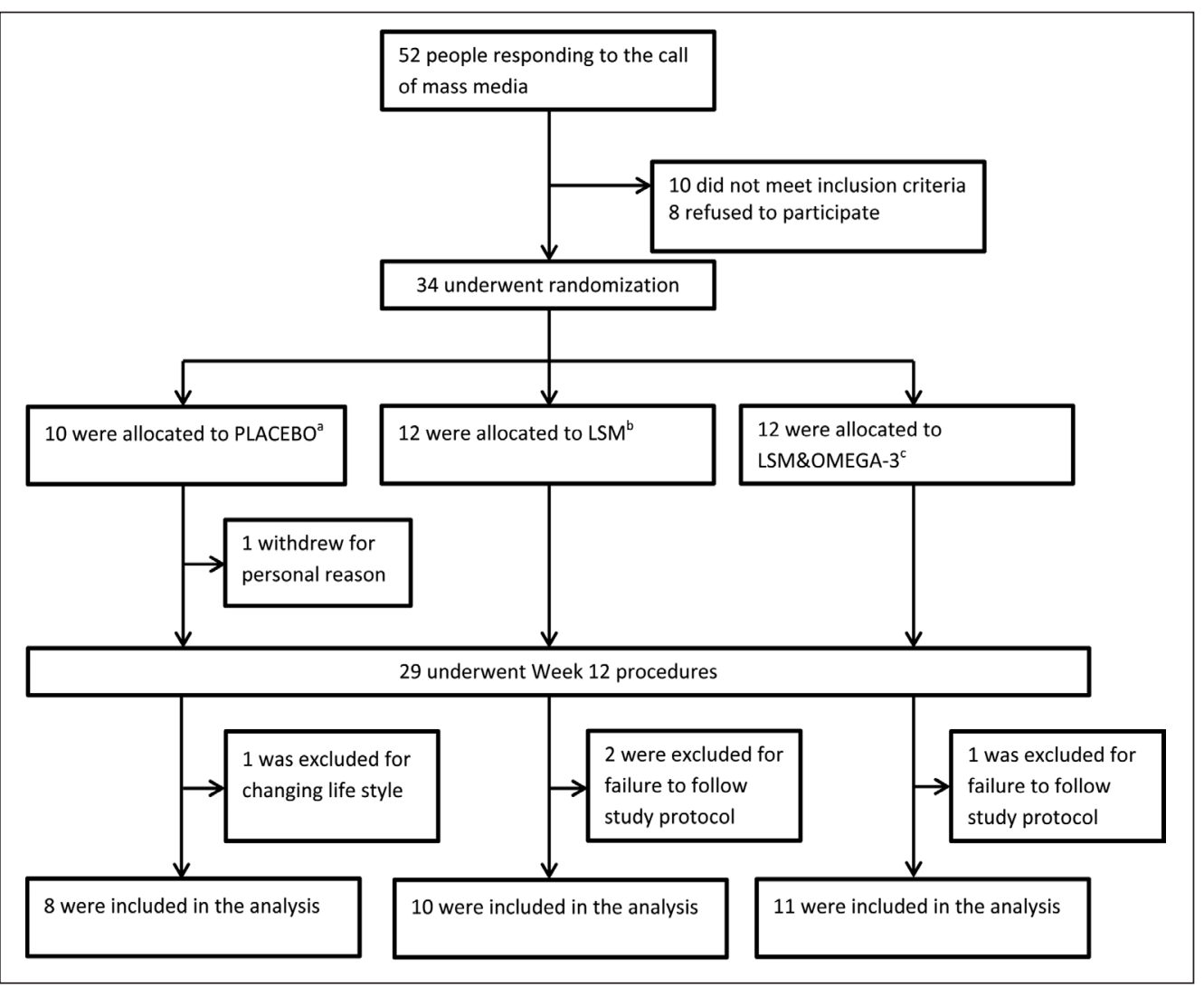

Fig. 1. Study design.

${ }^{\mathrm{a} G r o u p ~ w i t h ~ p l a c e b o ~ w i t h o u t ~ o t h e r ~ i n t e r v e n t i o n ~}$

${ }^{b}$ Group with placebo and managed physical activity

'Group with n-3 PUFA and managed physical activity

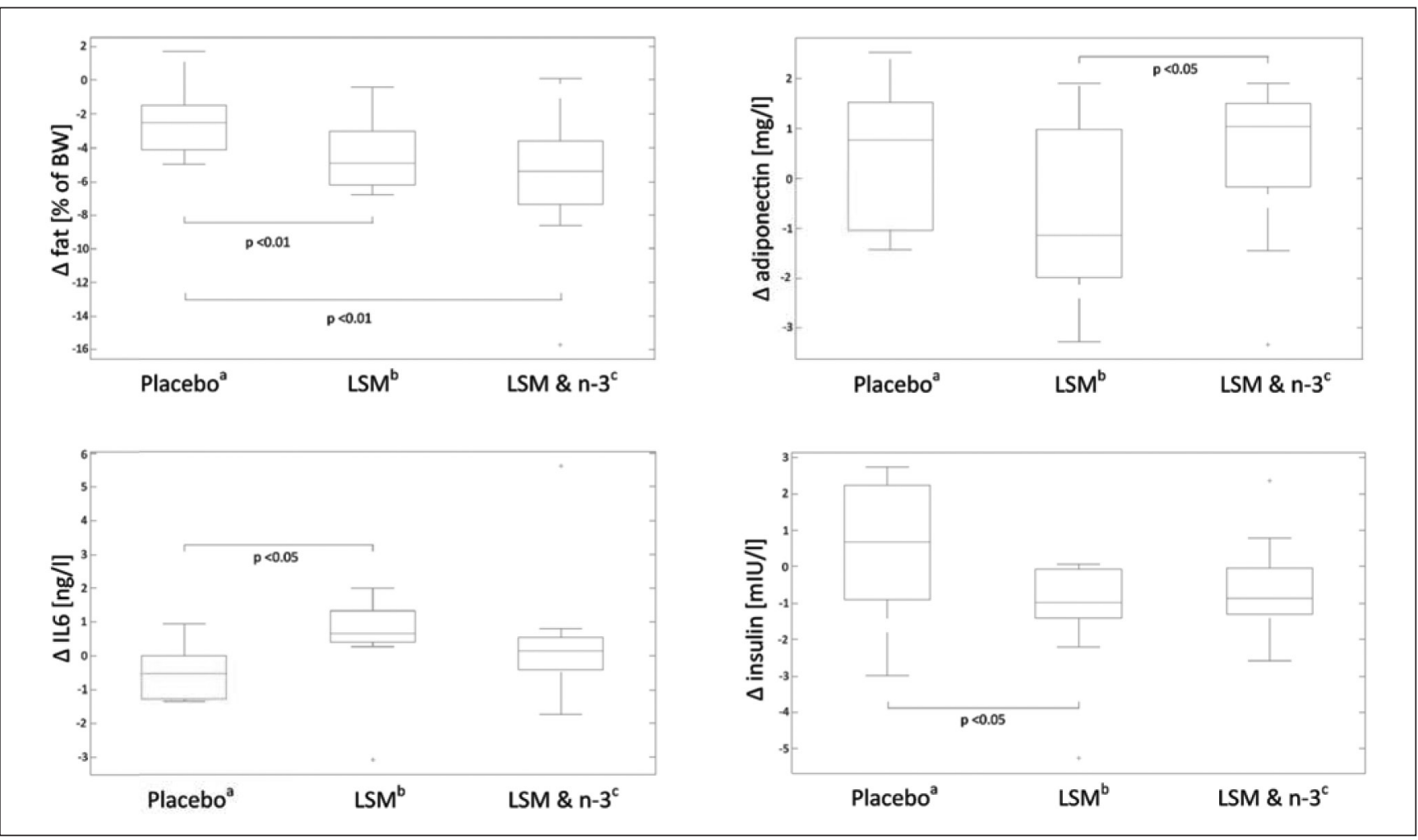

Fig. 2. Effects of interventions on body fat, expressed as \% of body weight, adiponectin, IL 6, and insulin.

$\Delta=$ changes between week 13 and the baseline, significance threshold for $p$ values is 0.05

aPlacebo without defined physical activity and nutrition

${ }^{b}$ Placebo with defined physical activity and nutrition

${ }^{\mathrm{n}} \mathrm{n}-3$ polyunsaturated fatty acids with defined physical activity and nutrition 
Table 2. Nutritional characteristic of diet before and after study

\begin{tabular}{|l|c|c|c|c|c|c|c|}
\hline \multirow{2}{*}{} & \multirow{2}{*}{ Unit } & \multicolumn{3}{|c}{ Before } & \multicolumn{3}{c|}{ After 12 weeks } \\
\cline { 3 - 8 } & & LSM & LSM \& omega 3 & Placebo & LSM & LSM \& omega 3 & Placebo \\
\cline { 3 - 8 } & & Mean \pm SD & Mean \pm SD & Mean \pm SD & Mean \pm SD & Mean \pm SD & Mean \pm SD \\
\hline Energy & $\mathrm{kcal}$ & $1,663.4 \pm 345.3$ & $1,732.5 \pm 480.5$ & $1,408.2 \pm 231.4$ & $1,683.3 \pm 403.2$ & $1,740.9 \pm 432.2$ & $1,698.1 \pm 588.1$ \\
\hline Protein/kg & $\mathrm{g} / \mathrm{kg}$ & $0.99 \pm 0.3$ & $1.08 \pm 0.2$ & $0.84 \pm 0.2$ & $1.03 \pm 0.3$ & $1.27 \pm 0.4$ & $0.96 \pm 0.4$ \\
\hline Protein & $\mathrm{g}$ & $75.0 \pm 22.0$ & $78.3 \pm 13.7$ & $65.1 \pm 17.0$ & $74.9 \pm 16.6$ & $85.6 \pm 27.6$ & $75.2 \pm 33.4$ \\
\hline Fat & $\mathrm{g}$ & $62.3 \pm 17.9$ & $61.5 \pm 24.1$ & $44.7 \pm 17.0$ & $62.7 \pm 29.6$ & $67.6 \pm 18.5$ & $68.9 \pm 25.9$ \\
\hline SFA & $\mathrm{g}$ & $24.2 \pm 9.1$ & $23.4 \pm 7.5$ & $16.9 \pm 8.1$ & $23.4 \pm 10.2$ & $24.7 \pm 7.9$ & $26.2 \pm 13.2$ \\
\hline MUFA & $\mathrm{g}$ & $19.6 \pm 6.6$ & $18.5 \pm 8.1$ & $12.8 \pm 8.1$ & $18.0 \pm 8.8$ & $19.6 \pm 7.7$ & $24.3 \pm 10.1$ \\
\hline PUFA & $\mathrm{g}$ & $11.5 \pm 8.1$ & $13.3 \pm 10.3$ & $10.2 \pm 3.4$ & $14.2 \pm 12.3$ & $16.6 \pm 10.3$ & $11.1 \pm 5.4$ \\
\hline Carbohydrates & $\mathrm{g}$ & $186.3 \pm 42.8$ & $195.9 \pm 64.5$ & $177.0 \pm 28.0$ & $191.8 \pm 39.3$ & $182.7 \pm 63.1$ & $179.4 \pm 59.1$ \\
\hline M-Di-Sacch & $\mathrm{g}$ & $60.4 \pm 22.6$ & $50.5 \pm 31.9$ & $41.6 \pm 10.8$ & $57.9 \pm 17.0$ & $52.8 \pm 30.7$ & $57.5 \pm 18.9$ \\
\hline Diet fiber & $\mathrm{g}$ & $17.3 \pm 10.7$ & $19.7 \pm 4.9$ & $17.8 \pm 6.2$ & $18.4 \pm 3.7$ & $21.7 \pm 7.5$ & $19.4 \pm 5.6$ \\
\hline Energy fat & $\%$ & $33.5 \pm 6.5$ & $31.8 \pm 6.2$ & $28.2 \pm 7.4$ & $32.3 \pm 9.6$ & $35.0 \pm 5.8$ & $36.1 \pm 6.7$ \\
\hline Energy protein & $\%$ & $18.4 \pm 3.7$ & $19.4 \pm 4.3$ & $18.7 \pm 2.2$ & $18.6 \pm 3.8$ & $20.5 \pm 4.9$ & $17.6 \pm 3.3$ \\
\hline Energy saccharides & $\%$ & $46.1 \pm 7.5$ & $46.3 \pm 7.2$ & $52.2 \pm 8.8$ & $47.8 \pm 8.8$ & $43.1 \pm 9.0$ & $44.4 \pm 9.5$ \\
\hline
\end{tabular}

LSM - group with placebo and managed physical activity

LSM \& omega 3 - group with n-3 PUFA and managed physical activity

SD - standard deviation

SFA - saturated fatty acids

MUFA - monounsaturated fatty acids

PUFA - polyunsaturated fatty acids

M-Di-Sacch - mono- + disaccharides

Table 3. Physical activity during study

\begin{tabular}{|l|c|c|c|c|}
\hline & Unit & LSM \& omega 3 & LSM & Placebo \\
\hline \multirow{2}{*}{ Physical activity } & Steps per day & $12,034 \pm 3,125$ & $10,118 \pm 1,984$ & Unmeasured \\
\cline { 2 - 5 } & MET min per week & $1,361 \pm 569$ & $1,021 \pm 405$ & Unmeasured \\
\hline
\end{tabular}

LSM \& omega 3 - group with n-3 PUFA and managed physical activity

LSM - group with placebo and managed physical activity

$\mathrm{MET}$ - metabolic equivalent of task

Table 4. Changes $(\Delta)$ between the end of study (week 13) and at the baseline

\begin{tabular}{|l|c|c|c|c|}
\hline \multirow{2}{*}{} & \multirow{2}{*}{ Units } & Placebo & LSM & LSM \& n-3 \\
\cline { 3 - 5 } & & $\Delta$-values & $\Delta$-values & \multicolumn{1}{c|}{-values } \\
\hline $\mathrm{n}$ & - & 8 & 10 & $-4.2^{\S}(-5.68 ;-0.6)$ \\
\hline Body weight & $\mathrm{kg}$ & $-0.05^{\star \S}(-2.1 ; 0.01)$ & $-3.25^{\star}(-5.8 ;-0.6)$ & $-1.4^{\S}(-2.32 ;-0.32)$ \\
\hline Body mass index & $\mathrm{kg} / \mathrm{m}^{2}$ & $-0.05^{\star \S}(-0.075 ; 0)$ & $-1.25^{\star}(-2 ;-0.2)$ & $-5^{\S}(-6.75 ;-2.5)$ \\
\hline Waist circumference & $\mathrm{cm}$ & $-1^{\star \S}(-2.5 ;-0.5)$ & $-7^{\star}(-8 ;-3)$ & $3.69^{\S}(1.14 ; 6.43)$ \\
\hline VO ${ }_{2}$ max & $\mathrm{L} / \mathrm{min}$ & $1.09^{\star}(-0.96 ; 3.25)$ & $3.97^{\star}(-1.44 ; 4.75)$ & $-3.9(-8.5 ; 0.63)$ \\
\hline Leptin & $\mu \mathrm{g} / \mathrm{L}$ & $0.72(-3.9 ; 2.38)$ & $-4.34(-6.35 ;-1.92)$ & $0(-101.23 ; 37.62)$ \\
\hline TNF a & $\mathrm{ng} / \mathrm{L}$ & $3.21(-38.41 ; 58.53)$ & $23.13(-55.36 ; 53.44)$ & $-0.83(-4.49 ; 2.13)$ \\
\hline IL8 & $\mathrm{ng} / \mathrm{L}$ & $1.76^{\star}(-1.77 ; 6)$ & $-3.71^{*}(-7.53 ; 1.86)$ & $0.048(-0.13 ; 0.3)$ \\
\hline Follistatin & $\mu \mathrm{g} / \mathrm{L}$ & $-0.01(-0.36 ; 0.13)$ & $0.021(-0.32 ; 0.45)$ & $-10.31(-71.32 ; 71.39)$ \\
\hline LIF & $\mathrm{ng} / \mathrm{L}$ & $26.46(-25.02 ; 88.11)$ & $2.85(-18.22 ; 169.57)$ & $-93.63(-174.5 ;-24.25)$ \\
\hline BDNF & $\mu \mathrm{g} / \mathrm{L}$ & $-87.91(-131.6 ;-35.31)$ & $-94.46(-268.61 ;-9.21)$ & $9.18(3.2 ; 11.15)$ \\
\hline Vitamin D & $\mu \mathrm{g} / \mathrm{L}$ & $8.26(10.88 ; 3.46)$ & $8.87(1.51 ; 11.19)$ & \\
\hline
\end{tabular}

Data are medians (value of the first quartile; third quartile). Statistical analysis was performed using a Wilcoxon's two-sided rank-sum test. ${ }^{*} p<0.05$ (LSM vs. placebo); $s_{\mathrm{p}}<0.05$ (LSM \& $n-3$ vs. placebo); ${ }^{\times} \mathrm{p}<0.05$ (LSM vs. LSM \& $n-3$ )

LSM - group with placebo and managed physical activity

LSM \& n-3 - group with n-3 PUFA and managed physical activity

$\mathrm{VO}_{2} \mathrm{max}$ - maximal oxygen consumption

TNF $\alpha$ - tumor necrosis factor $\alpha$

LIF - leukocyte inhibitory factor

BDNF - brain-derived neurotrophic factor 
acids. Decreased adiponectin and increased IL6 are important characteristics for the development of obesity related pathologies, such as type 2 diabetes, stem partly from dysregulated adipose tissue function, especially from its increased infiltration by macrophages and development of obese inflammatory phenotype (21, 22). Although the meta-analysis of randomized controlled trials (RCTs) showed that fish oil, naturally containing EPA+DHA, increases the circulating adiponectin in dose-dependent manner in humans; there was also unexplained heterogeneity, with a conclusion of no evidence for harm and support of possible benefits of n-3 fatty acid consumption on insulin sensitivity and adipocyte function (23). Recently using experiments in mice and overweight/obese type 2 diabetic patients the structures of novel members of fatty acid esters of hydroxy fatty acids were identified - lipokines derived from DHA and linoleic acid, present in serum and white adipose tissue after n-3 fatty acid supplementation, which exert beneficial anti-inflammatory properties while reducing macrophage activation by lipopolysaccharides (24). It was also documented that n-3 fatty acids may decrease the intensity of adipocyte-macrophage cross-talk to mitigate excessive adipose tissue macrophage inflammatory M1-polarization and inflammatory cytokine/chemokine secretion (25). Although from prospective long-term studies there has been demonstrated an inverse association between physical activity and inflammatory markers, particularly interleukin 6 (IL-6) (9), increased IL-6 and IL-8 levels were also reported following a 3 -day period of intense physical running training ( $2.5 \mathrm{~h} /$ day $)(26)$. Similarly, the transient dysfunction of the immune system, increased inflammation with a chronic increase in IL6, and oxidative stress are negative effects of physical exercise, which occurred in long-duration heavy training athletes (27). Thus, our results also help to clarify some benefits regarding the effects of $\mathrm{EPA}+\mathrm{DHA}$ on adiponectin and IL6 homeostasis in overweight women with moderate physical activity. As limitations of the study we consider only the short time (12 weeks) of intervention, small sample size, and the fact that the diet was not prepared in research kitchens but was only based in interventional arms on the instructions to the subjects and regular checking by nutritionists. The study was double blind only in part of LSM and LSM with supplementation. The placebo group was selected on individual decision not to adhere LSM and subject were significantly older than LSM or LSM \& n-3 groups. Moreover, there were included also women with postmenopausal status unlike the other two groups. The nature of the lifestyle intervention required disclosure for intervened participants and for part of care providers but was blind for statisticians and researchers who participated in the final evaluation and assessing outcomes. Due to external validity and applicability, our conclusions need to be further supported by results of other studies.

Our study is unique regarding the use of a complex lifestyle approach including the personalized diet and physical activity modification, as well EPA+DHA supplementation, which allowed us to demonstrate the additive improvements in adipose tissue function, and namely in immune response improvement, by active lifestyle modification in combination with EPA + DHA during weight loss process in women with overweight.

The results of our study document beneficial effects of a relatively low dose of $n-3$ fatty acid supplementation during the weight loss process using LSM in healthy women with overweight.
In healthy women with overweight in response to the combined intervention using LSM \& n-3, the adiponectin and IL6 levels were improved. Thus, women with overweight may be advised to increase their EPA+DHA intake, either in the form of dietary supplements or sea food and fish, in order to increase the efficacy of LSM on adipose tissue function, and to prevent diseases linked to inflammation.

\section{Acknowledgements}

This work was supported by Charles University in Prague, Faculty of Medicine in Pilsen grant: SVV-2014 - No 260055 and by the Charles University Research Fund (project number Q39). The work was also supported from ERDF under project "Research and Development of Intelligent Components of Advanced Technologies for the Pilsen Metropolitan Area (InteCom)" No. CZ.02.1.01/0.0/0.0/17_048/0007267. The study was approved by the Ethics Committee of the Faculty of Medicine and the University Hospital in Pilsen.

\section{Conflict of Interests}

None declared

\section{REFERENCES}

1. Yumuk V, Tsigos C, Fried M, Schindler K, Busetto L, Micic D, et al. European Guidelines for Obesity Management in Adults. Obes Facts. 2015;8(6):402-24.

2. Park YM, Myers M, Vieira-Potter VJ. Adipose tissue inflammation and metabolic dysfunction: role of exercise. Mo Med. 2014;111(1):65-72.

3. Hamasaki H. Daily physical activity and type 2 diabetes: a review. World J Diabetes. 2016;7(12):243-51.

4. Pasanisi F, Contaldo F, de Simone G, Mancini M. Benefits of sustained moderate weight loss in obesity. Nutr Metab Cardiovasc Dis. 2001;11(6):401-6.

5. Lindstrom J, Peltonen M, Tuomilehto J. Lifestyle strategies for weight control: experience from the Finnish Diabetes Prevention Study. Proc Nutr Soc. 2005;64(1):81-8.

6. Waxman A. WHO global strategy on diet, physical activity and health. Food Nutr Bull. 2004;25(3):292-302.

7. Wang PT, Chiang IT, Lin CY, Hou CW, Chen CY, Lee HH, et al. Effect of a two-month detraining on glucose tolerance and insulin sensitivity in athletes - link to adrenal steroid hormones. Chin J Physiol. 2006;49(5):251-7.

8. Aldred HE, Hardman AE, Taylor S. Influence of 12 weeks of training by brisk walking on postprandial lipemia and insulinemia in sedentary middle-aged women. Metabolism. 1995;44(3):390-7.

9. Hamer M, Sabia S, Batty GD, Shipley MJ, Tabak AG, Singh-Manoux A, et al. Physical activity and inflammatory markers over 10 years: followup in men and women from the Whitehall II cohort study. Circulation. 2012;126(8):928-33.

10. Sallam N, Laher I. Exercise modulates oxidative stress and inflammation in aging and cardiovascular diseases. Oxid Med Cell Longev. 2016;2016:7239639. doi: 10.1155/2016/7239639.

11. Kopecky J, Rossmeisl M, Flachs P, Kuda O, Brauner P, Jilkova Z, et al. n-3 PUFA: bioavailability and modulation of adipose tissue function. Proc Nutr Soc. 2009;68(4):361-9.

12. Ruan H, Dong LQ. Adiponectin signaling and function in insulin target tissues. J Mol Cell Biol. 2016;8(2):101-9.

13. Lee B, Shao J. Adiponectin and energy homeostasis. Rev Endocr Metab Disord. 2014;15(2):149-56.

14. Ito MK. Long-chain omega-3 fatty acids, fibrates and niacin as therapeutic options in the treatment of hypertriglyceridemia: a review of the literature. Atherosclerosis. 2015;242(2):647-56.

15. Flachs P, Rossmeisl M, Kopecky J. The effect of n-3 fatty acids on glucose homeostasis and insulin sensitivity. Physiol Res. 2014;63 Suppl 1:S93-118.

16. Suresh Y, Das UN. Long-chain polyunsaturated fatty acids and chemically induced diabetes mellitus. Effect of omega-3 fatty acids. Nutrition. 2003;19(3):213-28. 
17. Karvonen J, Vuorimaa T. Heart rate and exercise intensity during sports activities. Practical application. Sports Med. 1988;5(5):303-11.

18. Buckley JP, Borg GA. Borg's scales in strength training; from theory to practice in young and older adults. Appl Physiol Nutr Metab. 2011;36(5):682-92

19. Howley ET, Bassett DR Jr., Welch HG. Criteria for maximal oxygen uptake: review and commentary. Med Sci Sports Exerc. 1995;27(9):1292301.

20. Ortega JF, Morales-Palomo F, Fernandez-Elias V, Hamouti N, Bernardo FJ, Martin-Doimeadios RC, et al. Dietary supplementation with omega-3 fatty acids and oleate enhances exercise training effects in patients with metabolic syndrome. Obesity. 2016;24(8):1704-11.

21. Yu JG, Javorschi S, Hevener AL, Kruszynska YT, Norman RA, Sinha $\mathrm{M}$, et al. The effect of thiazolidinediones on plasma adiponectin levels in normal, obese, and type 2 diabetic subjects. Diabetes. 2002;51(10):296874.

22. Balistreri CR, Caruso C, Candore G. The role of adipose tissue and adipokines in obesity-related inflammatory diseases. Mediators Inflamm. 2010;2010:802078. doi: 10.1155/2010/802078.

23. Wu JH, Cahill LE, Mozaffarian D. Effect of fish oil on circulating adiponectin: a systematic review and meta-analysis of randomized controlled trials. J Clin Endocrinol Metab. 2013;98(6):2451-9.
24. Kuda O, Brezinova M, Rombaldova M, Slavikova B, Posta M, Beier P, et al. Docosahexaenoic Acid-Derived Fatty Acid Esters of Hydroxy Fatty Acids (FAHFAs) With Anti-inflammatory Properties. Diabetes. 2016;65(9):2580-90

25. De Boer AA, Monk JM, Liddle DM, Hutchinson AL, Power KA, Ma DW, et al. Fish-oil-derived n-3 polyunsaturated fatty acids reduce NLRP3 inflammasome activity and obesity-related inflammatory cross-talk between adipocytes and CD11b(+) macrophages. J Nutr Biochem. 2016;34:61-72.

26. Nieman DC, Luo B, Dreau D, Henson DA, Shanely RA, Dew D, et al. Immune and inflammation responses to a 3-day period of intensified running versus cycling. Brain Behav Immun. 2014;39:180-5.

27. Buonocore D, Negro M, Arcelli E, Marzatico F. Anti-inflammatory dietary interventions and supplements to improve performance during athletic training. J Am Coll Nutr. 2015;34 Suppl 1:62-7.

Received November 30, 2017 Accepted in revised form October 4, 2018 